\title{
Research on the Novel Cloud Pattern of Accounting based on Data Analysis and Mining under the Environment of Big Data and Could Computing
}

\author{
Liangcai Song 1 \\ ${ }^{1}$ Weifang University of Science and Technoloy, \\ Shouguang 262700, Shandong Province,China
}

\begin{abstract}
In this paper, we research on the novel cloud pattern of accounting based on data analysis and mining under the environment of big data and could-computing. Cloud instead of the traditional accounting information system will further speed up the enterprise information integration. Cloud accounting technique and method includes application software, application service platform and infrastructure capable of computation and data storage three levels. We discuss all the details in this paper. In the final part, we conclude the advantages and drawback of the cloud computing based technique and set up our prospect for further research.
\end{abstract}

Keywords: New Accounting Pattern; Data Analysis and Mining; Big Data and Could Computing.

\section{INTRODUCTION}

Cloud computing offers users the potential to reduce operating and capital expenses by leveraging the amortization benefits offered by large, managed infrastructures. As the change of social economic environment and the improvement of science and technology, managers begin to pay attention to the relevance of information, require companies to provide help to predict and evaluate the financial position and operating results of information in the future. The rapid development of cloud computing technology make the enterprise can use less cost to a more professional, more reliable, more efficient accounting information system. Cloud instead of the traditional accounting information system, will further speed up the enterprise information integration. Cloud accounting technique and method includes application software, application service platform and infrastructure capable of computation and data storage three levels. Cloud accounting of each layer consists of the corresponding service. Software as a service to build the cloud accounting, management, decision-making system, and other related system integration, delivered to the user through the network in the form of lease; Developers can every day many times for software upgrades, and these are transparent to the user; Users can completely break the limitation of space and time, at any time, any access to the Internet can place in a variety of ways to realize reimbursement, tax, auditing, remittances, such as remote work, truly realize the "mobile office". Platform as a service to build accounting information for the development of new application, new service platform, and cloud accounting database service, once the user has been the development and deployment of the application of complete, involved in the operation, management, monitoring the platform will be responsible for the work, the enterprise's financial data are unified management by the platform database services. Infrastructure as a service provides the virtualization infrastructure resources, in the form of a virtual machine to provide customers with the dynamic computing resources, implementing flexible storage capacity.

Cloud accounting application in the enterprise accounting information will has the following advantages. (1) Reduce the cost of accounting information application. Cloud to reduce accounting information application cost is reflected in three aspects: first, enterprises can 
rent IT infrastructure, from accounting and cloud service providers don't need to invest huge one-time IT, thoroughly eliminate the cost of purchase, installation, management resources; Second, enterprises in the use of these resources, you can pay according to the actual usage, and can be applied to the latest hardware and software resources; Third, the traditional accounting information system needs a lot of manpower and material resources to IT facilities maintenance and management, using the cloud accounting, hardware and software resources to more professional maintenance team, cost savings, and can achieve higher performance and reliability, enterprise can focus on strategic activity has long term effect to their own development. (2) Promote professional accounting information level. Cloud service provider employs professional accounting personnel and industry experts, they have a deep understanding of the business and have rich management experience, at the same time, research and development personnel with strong technical strength, and have a huge computing center to support cloud service. Even if companies in different industries can timely access to the business management the most targeted solutions. (3) Increase the reliability of the data management. Cloud accounting financial data in the cloud, have a professional team to help enterprise management information, a professional data centers to help enterprise backup data. Cloud computing is a large-scale distributed storage ways. (4) Improve the work efficiency. Look from within the enterprise, the future more economic transactions can be through the online funds transfer and electronic data interchange. When economic business happens, for example, data information through the Internet in a timely manner for processing in the cloud, the formation of the relevant accounting information, and can be further cost control, budget control, etc. Cloud accounting powerful computing ability, can form various indicators and statements, real-time managers to quickly understand the operating conditions, identify business risk. Therefore, to enhance the current pattern of cloud accounting, we research on the novel cloud pattern of accounting based on data analysis and mining under the environment of big data and could computing in this paper which will be discussed in detail in the following sections.

\section{The Core Technique of the Proposed Method}

The Principles of Cloud Computing. Cloud computing as a new product of the information society after a short span of 10 years or so has become a strong driving force leading the world change. But if no advanced technology, to provide data and information is of no use, can't meet the needs of users and then everything will be useless. Investigate the root cause, implement effective organization and management of data, is the important link to realize everything else, is also the core and important step. Cloud computing concept and service mode. Cloud computing is distributed processing, parallel processing and network to the further development of the processing. Users only need to rent the cloud computing services, only need to be installed on the terminal access to the Internet, the client browser can enjoy from the underlying storage cloud computing to the software through the network of a complete set of technical support services. In the figure one we show the structure. 


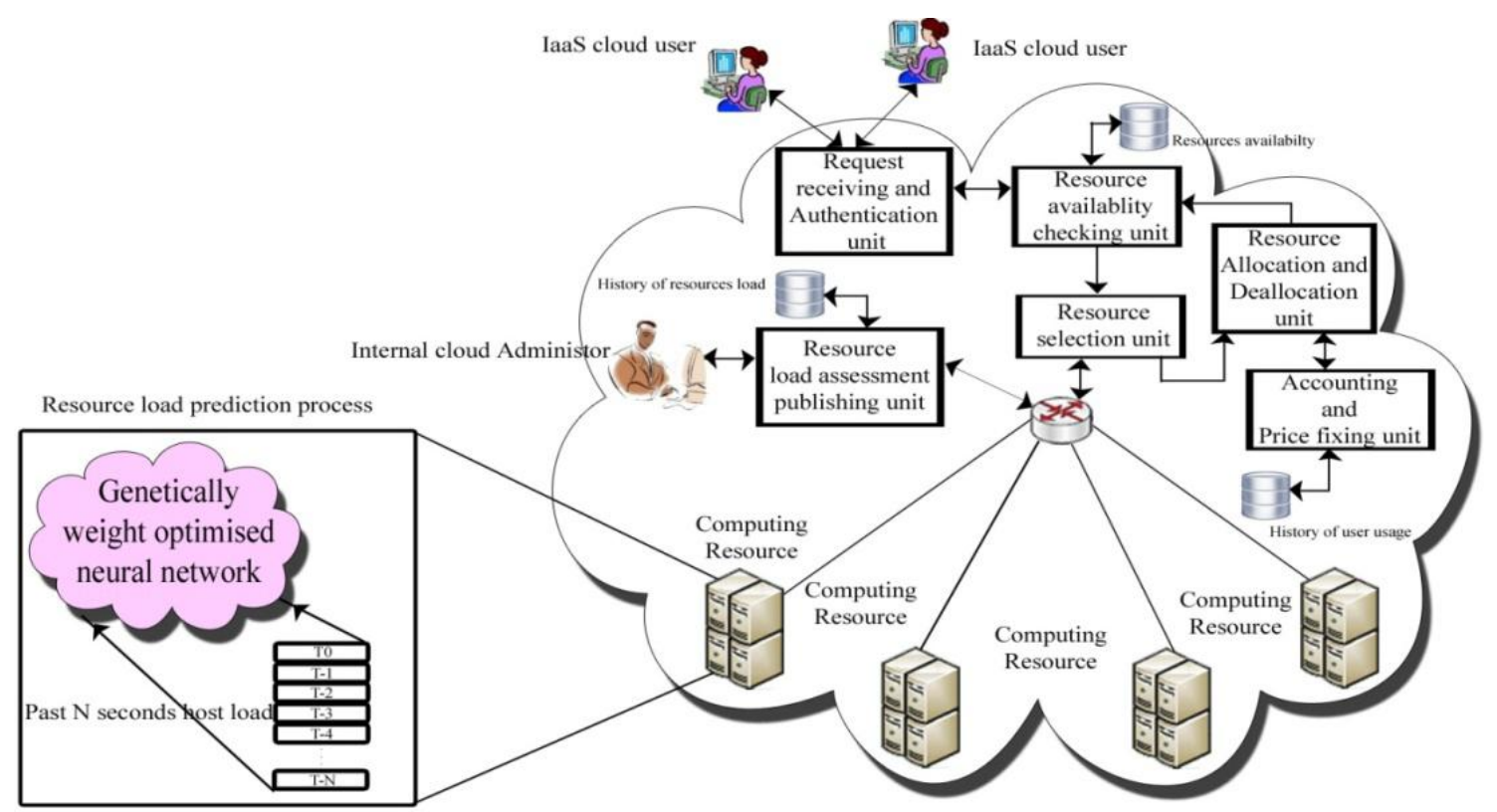

Fig 1. The General Structure of Cloud Computing Devices

Cloud computing services include three concepts: infrastructure as a service, platform as a service and software as a service. Infrastructure as a service refers to the cloud service providers to provide users with the required infrastructure, such as servers, operating system, database and network services, etc. Platform as a service refers to the cloud service providers to provide users with software development platform, allows users to fill in a platform the platform on the basis of the operation and upgrade. Software as a service refers to the cloud service providers to provide users with related industry software, for the use of the user. Also the provider shall be responsible for the software maintenance and upgrade. Therefore data warehouse and data mining technology has also been rapid promotion and further studies. Simply summed up as the data warehouse, data warehouse is a process, it decision makers to provide data for the enterprise, the data in the traditional operational database are hard to find. It has a subject oriented, do not update, large amount of data and data standardization. Therefore, combining cloud with accounting is necessary.

The Novel Cloud Pattern of Accounting. Look from the enterprise, the enterprise can always upward downstream enterprises, customers and partners and provide data. Around the current tax system gradually, for example, the cloud computing system platform is introduced into the tax information construction, enterprise can through the platform for the various tax operations; Certified public accountants can through the network to the enterprise's financial situation in a timely manner to make electronic audit report; Purchase and sale business contract in the form of the electronic data on the Internet to interact, to shift money via the Internet. Cloud accounting through Internet real-time processing enterprise with external authorities between finance and accounting business and accelerate the speed which improve the working efficiency. In industry, cloud providers already make use of pattern based descriptions and provide vendor-specific pattern formats and graphical notations. In the following figure, we show the example pattern. 


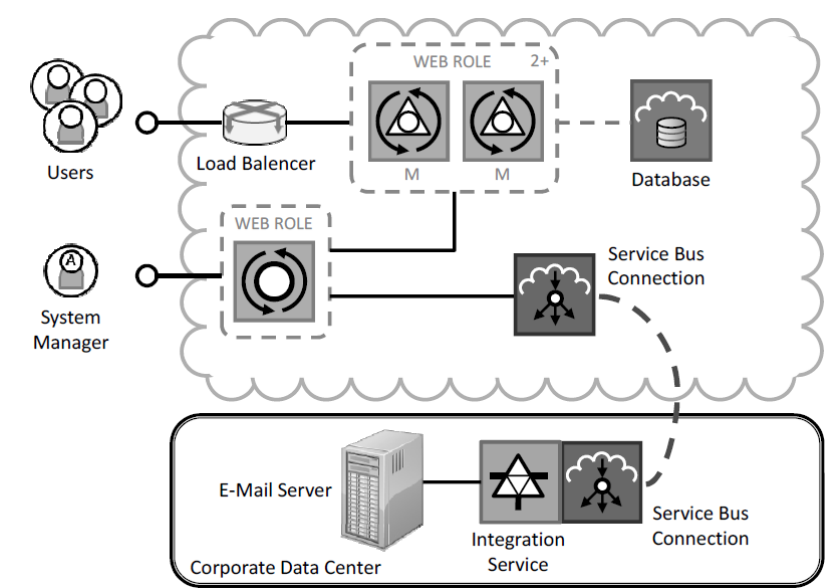

Fig 2. The General Exemplary Scenario Modeled for Cloud Accounting

First of all is the infrastructure layer virtual service cloud. Server and disk array through virtualization technology abstract into a cluster, can free combination of resources by the virtual operation management module is responsible for the unified management of all resources, according to the actual needs to allocate resources. Virtual cloud infrastructure on the system security, reliability and performance requirements is very high, usually provided by the big operators or large companies, such as China mobile and China telecom. The second is the platform service cloud. Platform service cloud on the one hand, focusing on enterprise data mining, including the extract, transform, and load data and information, etc., for the upper application provide highly efficient and stable data access channel, on the other hand also provides unified enterprise rights management ability. Through an open interface, the platform can carry a variety of business applications. Platform service cloud providers need to have a deeper understanding of the enterprise business data processing, at the same time also needs to have strong ability of the technical architecture. Located in the top of the cloud computing is a business service, cloud the layer is mainly made of different enterprise software provider, using the platform service provided by the cloud data ability, develop business applications of highly specific, through unified portal to provide corporate customers, meet the needs of different department of an enterprise, including the common ERP in the small and medium-sized enterprise accounting information, AIS and SCM system, etc. Due to the lower PAAS and IAAS structure based services, applications do not need to consider the underlying data operation and the management of the basic resources such as servers, storage, only need to focus on the actual business, so can more quickly with customized applications in different industry, and rapid deployment. In addition, because the upper application according to different customers on-demand using cloud resources, also can greatly save cost for customer.

The Challenges and Difficulties. Cloud computing model not only make the enterprise from the respective establishment, maintenance, management accounting information system, users can also can undertake various applications anytime, anywhere, for small and medium-sized enterprises information degree is low, the cloud computing model has obvious advantages, but there are also some problems. Small and medium-sized enterprises only less data needs to manage, but that is the lifeblood of them, and others don't want to mess up their data. "At present, the market economy in our country universal existence" the good faith crisis ", leading to many domestic enterprises are reluctant to put their own financial data, customer data such as core confidential information stored in the server of others. This is the cloud computing model free software installation and anywhere login directly through the Internet accounting information system for business processing and data storage custody attitudes. In strong extensibility of cloud computing mode, cloud computing provides software suppliers through specialization and economies of scale to reduce service costs at the same time, relying on large number of users to improve their economic benefits. The formation of the customer base depends on the high quality software service suppliers. And to provide a consistent set of small and medium-sized enterprise users with actual accounting 
information system, a lot of preparation work, mainly to comprehensive analysis of the needs of users. Different from traditional on-demand customized software, the cloud computing provider requirements can satisfy the different users' demand and different regions and different business rules, so the service of adaptability, expansibility and flexibility requirement is very high, also has a higher demand on the technology. Finally, security concern is common for the cloud service users, how to enhance the secure the data security is a challenge for us researchers.

\section{CONCLUSIONS}

In this paper, we research on the novel cloud pattern of accounting based on data analysis and mining under the environment of big data and could-computing. Cloud computing model not only makes the enterprise from the respective establishment, maintenance, management accounting information system but users can also can undertake various applications anytime as well. Through an open interface, the platform can carry a variety of business applications. Platform service cloud providers need to have a deeper understanding of the enterprise business data processing. Therefore, leading could service to accountant is a great choice to enhance the performance of the business.

\section{References}

[1] McGuire M P, Roberge M C, Lian J. HydroCloud: A Cloud-Based System for Hydrologic Data Integration and Analysis[C]. //International Conference on Computing for Geospatial Research \& Application. IEEE, 2014:9 - 16.

[2] Wang Y, Wang L, Liu H, et al. Large-Scale Clinical Data Management and Analysis System Based on Cloud Computing[J]. Lecture Notes in Electrical Engineering, 2014:1575-1583.

[3] Yang W P, Duan D Q. Architecture of Cloud-Based Crime Spatial Data Mining Platform[J]. Mechatronics Engineering \& Modern Information Technologies in Industrial Engineering, 2015.

[4] Craus M, Butincu C. The Potential of Cloud Computing for Analysis and Finding Solutions in Disasters[J]. Nato Science for Peace \& Security, 2014:239-252. 